\title{
The E3 Ubiquitin Ligase NARF Promotes Colony Formation in vitro and Exhibits Enhanced Expression Levels in Glioblastoma Multiforme in vivo
}

\author{
Tucker W. Anderson, Christopher Wright, and William S. Brooks \\ Department of Biology \\ Freed-Hardeman University \\ Henderson, Tennessee
}

Received: July 2, $2010 \quad$ Accepted: November 9, 2010

\begin{abstract}
The ubiquitin ligase NARF is a newly identified protein belonging to a small family of structurally similar E3 proteins. NARF is a negative regulator of the canonical Wnt- $\beta$-catenin pathway, targeting TCF/LEF family members for proteolytic degradation through poly-ubiquitination. We examined the role that NARF plays in cell division and found that overexpression of NARF in a colony forming assay increases colony formation in a RING finger-dependent manner. Furthermore, we demonstrate that NARF transcripts are expressed at a higher level in the grade IV brain tumor glioblastoma multiforme as compared with low grade astrocytomas. Our data thus indicate that NARF is a positive regulator of cell growth and may be involved in the tumorigenic process.
\end{abstract}

\section{INTRODUCTION}

Eukaryotic cells employ a range of post-translational protein modifications to serve as signals for a diversity of cellular processes. These modifications can alter protein shape, activity, function, localization, stability, etc. Included in this large group of post-translational modifications is protein ubiquitination. Ubiquitin is a small, highly conserved 76 amino acid protein that is covalently linked to substrate proteins by a three-step enzymatic reaction (reviewed in [1]). First, the ubiquitin activating enzyme, or E1, binds ubiquitin in an ATP-dependent manner through a cysteine residue to form a thio-ester bond with ubiquitin. Second, the now activated ubiquitin peptide is transferred onto the conserved cysteine residue of one of several ubiquitin conjugating enzymes, or E2s, once again forming a thio-ester bond between the E2 and ubiquitin. The final step of this process, the covalent attachment of ubiquitin onto a lysine residue of the substrate protein, involves the action of a ubiquitin ligase, or E3 enzyme. While there are known to be three different protein domains which can serve as ubiquitin ligase domains, the most common is the RING (Really Interesting New Gene) finger domain [2]. RING finger ubiquitin ligase proteins function as facilitators in the transfer of ubiquitin peptides from the E2 enzyme onto the substrate protein by bringing substrate proteins into close proximity with the ubiquitin conjugating enzyme allowing for direct transfer of the ubiquitin molecule onto a specific lysine residue of the substrate [3].

Once a substrate protein is ubiquitinated, this cascade of events may be repeated several times to form a polyubiquitin chain. A chain consisting of four or more ubiquitin peptides linked through the lysine-48 and glycine-78 residues of consecutive ubiquitins serves as a signal for proteolytic degradation by the $26 \mathrm{~S}$ proteasome. Ubiquitination, however, is not restricted to serve as just a signal for protein destruction. It has been shown in recent years to also serve a variety of other purposes including altering both localization as well as biochemical activity of a protein $[4,5]$. These functions, though, most often result from protein mono-ubiquitination or 


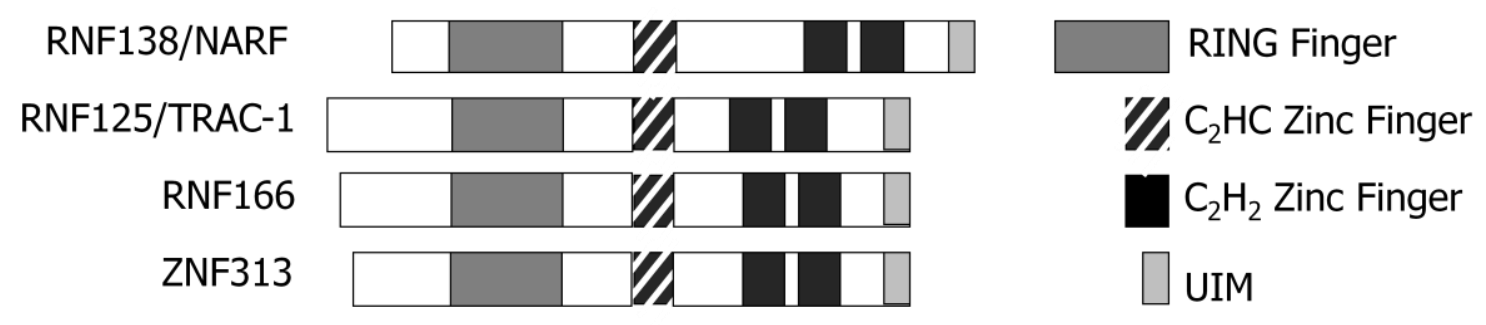

Figure 1. Domain structure comparison of NARF and related family proteins. RNF138/NARF, RNF125/TRAC-1, RNF166, and ZNF313 domain structures are shown. Each protein contains a RING domain near its amino-terminus, a central $\mathrm{C}_{2} \mathrm{HC}$ zinc finger and two $\mathrm{C}_{2} \mathrm{H}_{2}$ zinc fingers, as well as a ubiquitin interacting motif (UIM) at its $\mathrm{C}$-terminus. Each protein shares $\sim 40 \%$ similarity at the protein level.

poly-ubiquitin chains utilizing one of several other lysine residues in the ubiquitin protein sequence

E3 ubiquitin ligases are the most numerous and diverse of those enzymes involved in the ubiquitination cascade. Hundreds of ubiquitin ligase proteins are encoded by the human genome with more than 600 RING domain ligases alone being present [2]. This is in contrast to the single human E1 gene and the approximately 35 human E2 genes [6]. Therefore, in the ubiquitination cascade it is the ubiquitin ligase which confers substrate specificity. Each ubiquitin ligase would be expected to modify one or a few substrate proteins, augmenting their stability, function, or localization. The need for a great number of distinct E3 enzymes is highlighted by the ever increasing number of proteins known to be modified by either mono- or polyubiquitination.

Nemo-like kinase (NLK)-associated ring finger protein (NARF), originally known as RNF138, is a 245 amino acid protein with RING finger-dependent ubiquitin ligase activity [7]. A previous publication has demonstrated that this protein is associated with nemo-like kinase (NLK) and cooperates with the kinase to down-regulate TCF/LEF transcriptional activity by poly-ubiquitinating TCF/LEF, targeting it for proteasomemediated destruction [7]. Through this activity, NARF functions as a negative regulator of the Wnt- $\beta$-catenin signaling pathway.

NARF is one member of a family of 4 RING ubiquitin ligases, which also includes RNF125/TRAC-1, RNF166, and RNF114/ZFP313 [8]. Each of these proteins contains an amino-terminal RING finger domain, three central $\mathrm{C}_{2} \mathrm{HC}$ or $\mathrm{C}_{2} \mathrm{H}_{2}$ zinc fingers and a carboxy-terminal ubiquitin binding motif (UIM) (Figure 1). The $\mathrm{C}_{2} \mathrm{H}_{2}$ zinc finger is found in many transcription factors and often binds directly to dsDNA [9]. Some $\mathrm{C}_{2} \mathrm{H}_{2}$ fingers, however, bind to RNA or to other proteins making this domain quite versatile in function. The UIM has been shown to bind ubiquitinated proteins in a manner analogous to an $\mathrm{SH} 2$ domain binding to phosphorylated proteins [10]. While sharing a conserved domain structure, the limited homology of these proteins $(\sim 40 \%$ similarity) and differences in expression indicate that they likely play diverse roles in cell physiology. The RNF125/TRAC-1 protein has been shown to be a membrane-bound molecule which positively regulates T-cell activation, downregulates RIG-I antiviral signaling, and suppresses HIV-1 replication [8, 11-12]. RNF114/ZFP313 is a psoriasis susceptibility gene [13], while nothing is known yet about the function of RNF166.

In the current study, we have examined the effects of NARF overexpression on cell division. In vitro colony forming assays have demonstrated an increase in colony formation in response to overexpression of exogenous NARF. This increase in colony formation is RING finger-dependent. Furthermore, analysis of a previously conducted microarray experiment [14] has shown that there is a significant increase in NARF gene expression in glioblastoma multiforme (GBM) tumors over that in low grade astrocytomas (LGA). These results reveal a 
potential role for NARF in cell division and the tumorigenic process.

\section{MATERIALS AND METHODS}

\section{a. In vitro Ubiquitination Assay}

CDNA encoding wild type NARF, the NARF-CHA mutant, or GFP were subcloned into the pFlag-CMV10 plasmid (Sigma) using standard molecular biology techniques [15]. HEK293T cells were transiently trnsfected with the indicated plasmid using Lipofectamine 2000 (Invitrogen) according to manufacturer's instructions. Thirty-six hours following transfection, cells were harvested and lysed in Mammalian Cell Lysis Buffer prepared as described previously [16]. The lysates were cleared by centrifugation and protein yield was quantified using the Bradford assay. $1 \mathrm{mg}$ of whole cell lysate was immunoprecipitated with Flag-Agarose (Sigma) overnight at $4^{\circ} \mathrm{C}$. Following, agarose beads were washed twice with lysis buffer then twice with ubiquitination assay buffer (50mM Tris, pH 7.5, 2.5mM $\mathrm{MgCl}_{2}$, $0.5 \mathrm{mM}$ dithiothreitol, $300 \mu \mathrm{M}$ ATP). In vitro ubiquitination assays were performed by combining Flag-Agarose beads bound to the respective precipitated proteins with $100 \mathrm{ng}$ human E1 (Boston Biochem), $1 \mu \mathrm{g}$ of $\mathrm{UbcH} 1$ (Boston Biochem), $\mathrm{His}_{6}$-ubiquitin (Boston Biochem), $1 \mu \mathrm{g}$ of bacterial lysate, and $1 \mathrm{X}$ Energy Regeneration System (Boston Biochem) in reaction buffer. Reactions were carried out at $30^{\circ} \mathrm{C}$ for $1.5 \mathrm{~h}$ followed by quenching by boiling in an equal volume of $2 X$ protein sample buffer (4\% SDS, $10 \%$ glycerol, $\quad 0.01 \%$ bromophenol blue, $30 \mathrm{mg} / \mathrm{mL}$ dithiothreitol). Whole cell lysates and products of the in vitro assay were separated by a $6-12 \%$ gradient SDS-PAGE gel, transferred to a nitrocellulose membrane, and Western blotting was conducted using either an anti-His antibody (Amersham) or anti-Flag M2 antibody (Sigma).

\section{b. Colony Forming Assay}

CDNA encoding wild type NARF or the NARF-CHA mutant was subcloned into the pcDNA3.1HA plasmid using standard molecular biology techniques [15]. HeLa cells were transfected with the indicated plasmid using Lipofectamine 2000. Twenty- four hours later, the cells were trypsinized and counted using trypan blue and a hemocytometer. 25,000 cells were plated into each well of a 6 well plate and allowed to adhere for $24 \mathrm{~h}$. Cells were then treated with $500 \mu \mathrm{g} / \mathrm{mL}$ G418 (Sigma) in tissue culture media for 2 weeks. The media was changed every 3-4 days during this period. Following this period, cells were fixed in $3.7 \%$ paraformaldehyde in phosphate buffered saline (PBS) for $5 \mathrm{~min}$. $0.05 \%$ crystal violet was then added to stain cells for $30 \mathrm{~min}$. Plates were then washed with water and allowed to air dry. Replicative data were analyzed in Microsoft Excel and an unpaired student's $t$-test was performed to assess statistical significance at the 0.01 level.

\section{c. Gene Expression Analysis}

The European Bioinformatics
Institute Gene Expression Atlas 2.0 (http://www.ebi.ac.uk/gxa/) was queried for expression data for the human gene RNF138/NARF. Processed gene expression data were downloaded from the Gene Expression Atlas for the experiment numbered E-MEXP-567 and opened using Microsoft Excel. Expression data for each gene in each tumor sample were assembled and analyzed using MS Excel. Fold expression increases were calculated by dividing the average expression in all GBM tumors by the average expression in all LGA tumors [Avg GBM/Avg LGA]. Expression data in each sample were also normalized for comparison by dividing each expression quantity by the average of all LGA expression quantities for each respective gene. A paired student's t-test was performed with a significance level of 0.01 to determine statistical significance.

\section{RESULTS}

a. Overexpression of NARF promotes colony formation in a RING-dependant manner

Our laboratory is interested in examining new proteins which play roles in the cell cycle and tumorigenic processes. We began examining a potential role for the recently identified protein NARF [7] in these pathways. To this end, we generated wild 


\section{A}

NARE CPVCQEVLKTPVRTTACQHVECRKCELTAMRESGAHCPLC

NARE-CHA CPVCQEVLKTPVRTTAAQAVECRKCELTAMRESGAHCPLC
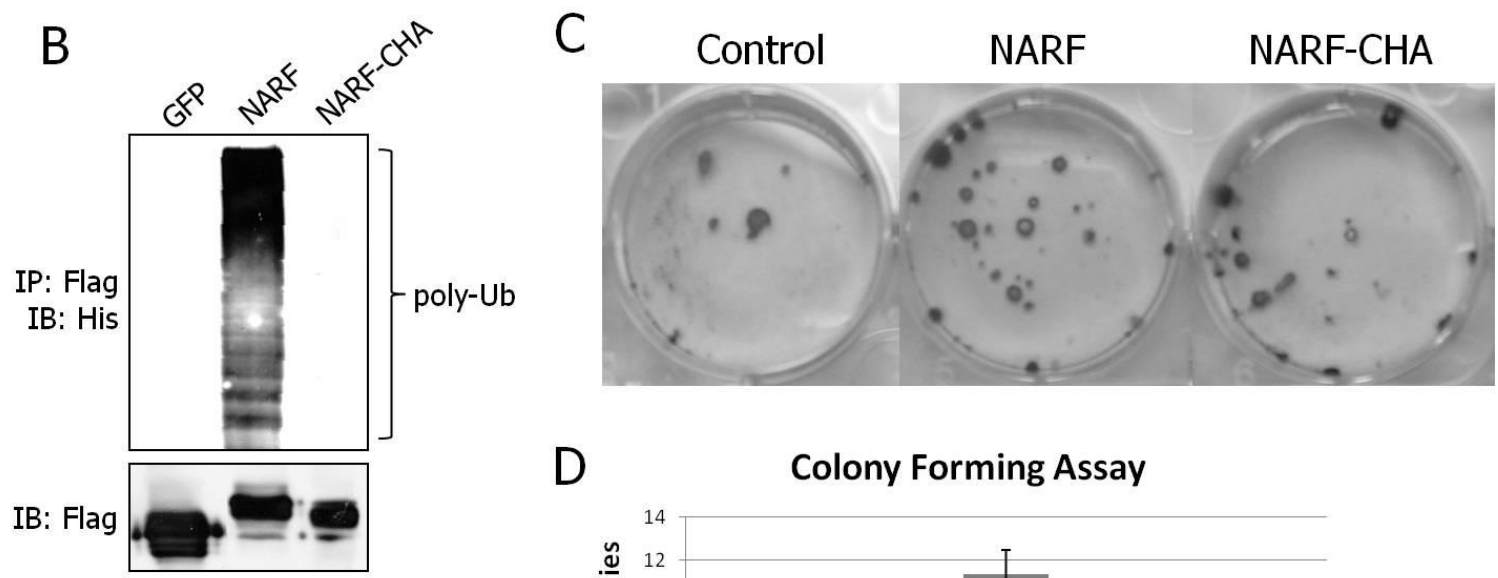

D

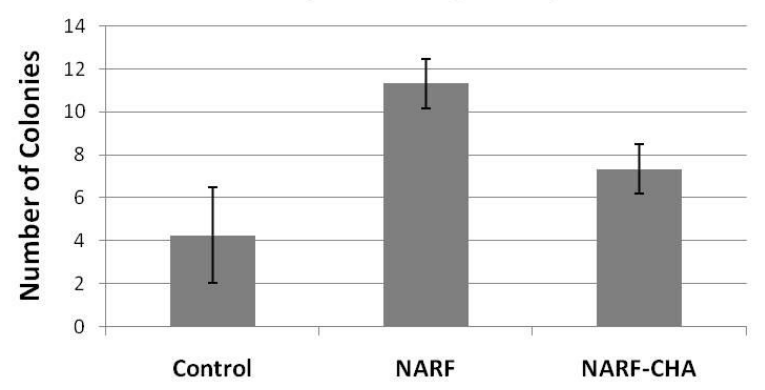

Figure 2. Overexpression of NARF promotes colony formation in a RING-dependent manner. A, A sequence alignment is shown between the RING domain of wild type NARF and NARF-CHA. Asterisks indicate the conserved zinc chelating residues and double asterisks indicate the mutated amino acids in NARF-CHA. B, Flag-tagged GFP, wild type NARF, and NARF-CHA were assayed for ubiquitin ligase activity in an in vitro ubiquitination assay followed by separation on a $6-12 \%$ gradient SDS-PAGE gel. Western blotting indicates that wild type NARF but not GFP or the NARF-CHA mutant is capable of functioning as an ubiquitin ligase. C, The indicated proteins were expressed in HeLa cells followed by stable selection using G418. The surviving colonies were stained with crystal violet and imaged for comparison. D, The results of 4 repeated colony forming assays were combined and the average number of colonies is plotted with standard deviations. A significant increase in colony number $(p<0.01)$ is seen with NARF overexpression but not with NARF-CHA overexpression.

type and RING-mutant NARF expression constructs in the pFLAG-CMV10 and pcDNA3.1HA parental plasmid vectors. zinc ions; this chelation is required for the proper structure of the domain. We mutated cysteine-34 and histidine-36 within the RING finger domain each to alanine and call this mutant NARF-CHA (Figure 2A). A similar NARF point mutant was generated previously and shown to lack ubiquitin ligase activity [6]. To confirm that our wild type construct was functional as a ubiquitin ligase and that the CHA mutant lacked ubiquitin ligase activity, we conducted an in vitro ubiquitin ligase assay using
RING domains share a conserved arrangement of 8 cysteine and histidine residues which cooperatively chelate two immunoprecipitated proteins. Flag-tagged NARF or NARF-CHA was transiently expressed in 293T cells and immunoprecipitated. We also transfected Flag-tagged green fluorescent protein (GFP) to serve as a negative control. Immunoprecipitated proteins were incubated with reaction components then separated by SDS-PAGE followed by Western blotting. The upper panel of Figure 2B demonstrates ubiquitin ligase activity for wild type NARF but not for GFP or NARF-CHA as 
demonstrated by the high molecular weight ladder of poly-ubiquitin bands. The lower panel is a Western blot of cell lysates from the transfected cells showing the expression of each protein prior to immunoprecipitation. We have routinely seen a slightly faster migration of the CHA mutant compared with the wild type protein. The predicted molecular weight of Flag-NARF is $31.2 \mathrm{kDa}$ while that of Flag-NARF-CHA is $31.1 \mathrm{kDa}$. This difference in size likely accounts for the minor variation in migration for the NARFCHA protein.

Following confirmation of the activities of each protein, a series of in vitro colony forming assays similar to those performed previously [17] were conducted to explore the effects of NARF overexpression on cell growth. HeLa cells were transfected with the pcDNA3.1HA/NARF, pcDNA3.1HA/NARF-CHA, and pcDNA3.1HA empty vector plasmids using a lipid carrier reagent (Lipofectamine 2000, Invitrogen). Following transfection, stable transfectants were selected for 2 weeks with the drug G418. The pcDNA3.1HA plasmid contains the neomycin resistance gene; therefore, those cells which stably integrated the plasmid would survive in the presence of this drug while those cells which did not would be killed. Following the selection period, cell colonies were fixed and stained with crystal violet. Figure $2 \mathrm{C}$ is a representative image of each plate of cells. A higher number of surviving colonies was seen in those cells stably overexpressing NARF as compared with the control and NARF-CHA transfectants. To quantify the data, four replicated experiments were performed, and the data were combined and are shown in Figure 2D. As seen, HeLa cells stably overexpressing wild type NARF are more competent to form colonies than control cells. Statistical analysis of the data using a student's $t$-test indicates that the increase is significant $(p<0.01, n=4)$. We saw a slight increase in colony formation with the NARF-CHA mutant over that of control cells, however statistical analysis indicated that the difference was not significant at the 0.01 level $(p=0.03, n=4)$. These data indicate that NARF overexpression can enhance cell growth and/or viability in a RING-dependant manner. b. NARF exhibits enhanced expression in Glioblastoma Multiforme tumors

Genes whose protein products enhance cell division and cell viability are frequently overexpressed in human tumors. Since elevated levels of NARF were able to increase HeLa cell growth in an in vitro colony forming assay, we hypothesized that the gene may be overexpressed in human tumors. To address this hypothesis, we searched the European Bioinformatics Institute Gene Expression Atlas (http://www.ebi.ac.uk/gxa) for gene expression data of NARF. This online resource contains a searchable repository of hundreds of diverse microarray experiment data. When queried for NARF expression data, numerous experiments were available for analysis. We chose to examine the expression of NARF in the highly lethal, grade IV brain tumor glioblastoma multiforme (GBM), experiment E-MEXP-567 [14]. In this particular microarray experiment, tumors extracted from patients with glioblastoma multiforme and low grade astrocytoma (LGA) were examined for gene expression profiles. We chose this particular set of data for our analysis because we were interested in comparing the expression of NARF between tumors of the highly malignant brain cancer glioblastoma with the much less malignant astrocytomas. Insights into what gene expression changes promote a more malignant tumor phenotype have value in understanding the tumorigenic process. Expression data for NARF as well as the housekeeping protein alpha-tubulin, cell cycle regulator cyclin $\mathrm{B} 1$, and mitotic enzyme topoisomerase lla were extracted from the repository and analyzed.

As seen in Figure $3 \mathrm{~A}$, there was no average increase in expression of the housekeeping gene $\alpha$-tubulin when comparing LGA tumors with GBMs. In contrast, both cyclin B1 and topoisomerase Ila were elevated in expression in GBM tumors. A 3.7 fold average increase in expression was observed with cyclin B1 and a 7.3 fold increase was seen in topoisomerase Ila. Both of these genes are known to play important roles in cell division, therefore an increase in their expression levels is not surprising. NARF displayed a 2.2 fold average increase in expression in 


\section{A Expression Increases between LGA and GBM}
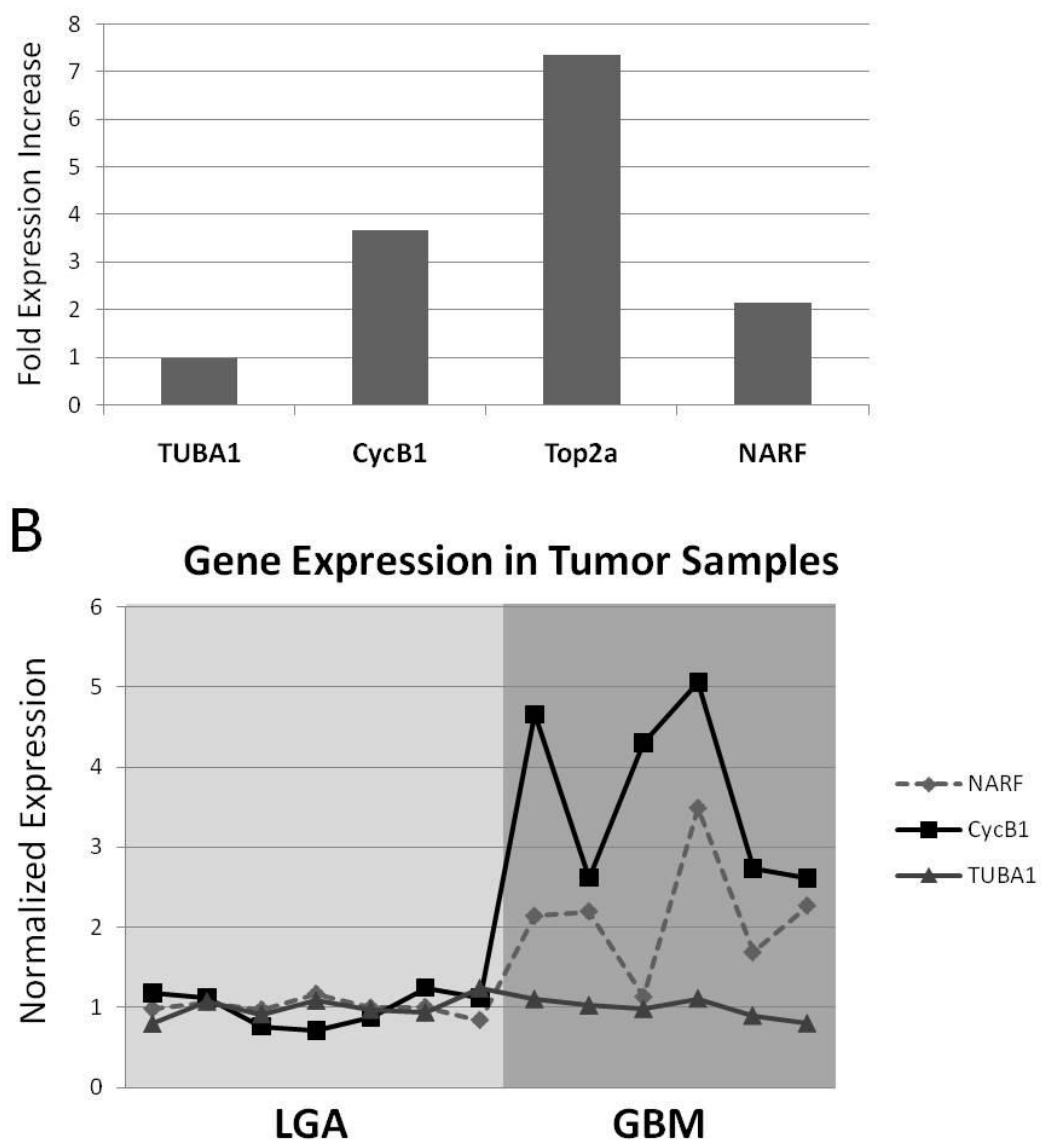

Figure 3. NARF is overexpressed in Glioblastoma Multiforme tumors as compared with Low Grade Astrocytomas. Gene expression data for the indicated genes were extracted from the Gene Expression Atlas experiment E-MEXP-567. A) The average fold expression increase in GBM tumors over LGA tumors is plotted. Fold expression increases were calculated by dividing the average expression in all GBM tumors by the average expression in all LGA tumors [Avg GBM/Avg LGA]. B) Normalized expression levels for each gene are plotted for each LGA and GBM tumor sample. Levels of $\alpha$-tubulin are unchanged in GBM tumors while levels of cyclin B1 and NARF are significantly elevated (NARF $p$-value $<0.01$ ).

GBM tumors over LGA tumors, indicating that its expression is also elevated in the more malignant brain tumor.

We plotted a graph of each gene's expression in individual tumor samples for further comparison (Figure 3B). Seven low grade astrocytoma tumors and six GBM samples were included in the study. The expression of each gene in each tumor was normalized for comparison by dividing the quantitated expression from each tumor sample with the average expression of the gene among all LGAs [expression/LGA avg expression]. This normalization allowed for a more direct comparison of expression increases for each gene in the GBM tumors. As shown, a-tubulin had no expression change in any of the GBM tumors allowing it to serve as a negative control gene. Cyclin B1 was elevated in each of the GBM tumors with a fold increase ranging from 2.6 to 5.0. NARF displayed an elevated expression in 5 of the 6 tumor samples with the highest increase measuring 3.5 fold. Topoisomerase lla was omitted from this graph because of its high expression increases (range 3.3 - 13.1). We conducted a paired student's t-test to determine if the 
increase in NARF expression in the GBM tumors was statistically significant as compared with that of $\alpha$-tubulin. NARF was significantly elevated with a $p$-value of 0.006 $(n=6)$. These data confirm the hypothesis that NARF is overexpressed in some human tumors and further supports the hypothesis that NARF is involved in the control of cell division and tumorigenesis.

\section{DISCUSSION}

NARF was previously identified in a screen for proteins that interact with the carboxy-terminus of Xenopus laevis nemolike kinase (NLK) [7]. It was further demonstrated that NARF could function as an ubiquitin ligase and ubiquitinated TCF/LEF in an NLK-dependent manner. We demonstrate that elevated expression of NARF in cultured HeLa cells can enhance colony formation. Furthermore, we have shown that like other genes involved in the cell division process, NARF is overexpressed in a portion of human malignancies.

NARF is one member of a family of RING finger proteins [8]. Both NARF and RNF125/TRAC-1 have been demonstrated to possess RING-dependent ubiquitin ligase activity [7]. While this has not been demonstrated for RNF166 or RNF114/ZFP313, the high degree of homology within the RING domain of each of these 4 proteins would indicate that they too are ubiquitin ligases. Each of these proteins share greater than $40 \%$ similarity at the amino acid level and $100 \%$ identity of zinc binding-cysteine and histidine residues within the RING and zinc fingers. ZNF313 was shown to be differentially expressed between the testes of fertile adults and those of azoospermic patients [18]. TRAC-1 was identified in a screen for novel regulators of T-cell receptor expression [10]. These proteins all contain three zinc finger motifs, one $\mathrm{C}_{2} \mathrm{CH}$ type and $2 \mathrm{C}_{2} \mathrm{H}_{2}$ type zinc fingers. This motif is one of the most abundant in eukaryotes and is known to bind DNA, RNA, as well as other proteins. Additional studies are required to determine the binding properties of the zinc fingers within this family of proteins. Zhao and colleagues have noted that a C-terminal truncation of TRAC- 1 resulted in a dominant negative effect on the expression of the TCR
[11]. This truncation eliminates the third $\mathrm{C}_{2} \mathrm{H}_{2}$ finger and UIM while leaving the first and second zinc finger intact. This effect was abolished when the RING domain was mutated in conjunction with the C-terminal truncation. No explanation was suggested to explain this dominant negative effect, but we propose that this phenotype is a result of deletion of this third $\mathrm{C}_{2} \mathrm{H}_{2}$ domain and/or the UIM. While NARF and ZNF313 show high mRNA expression in testis and much lower expression in other normal tissues ([18] and unpublished data), TRAC-1 displays an expression pattern consistent with a role in leukocyte function [11]. Moreover, its functional role in regulating TCR expression highlights this. It is thus likely that, although these protein share domain structures, they may have divergent, tissue-specific roles in mammalian physiology.

Yamada et al., demonstrated a role for NARF in the Wnt- $\beta$-catenin signaling pathway [7]. In their studies, NARF was found to interact directly with nemo-like kinase and poly-ubiquitinate TCF/LEF family transcription factors, thereby negatively regulating this signaling pathway. In human cancers abundant evidence supports a role for the canonical Wnt pathway in promoting tumor growth [19-20]. This pathway has been implicated in cell proliferation, survival, and tumor progression. A recent report has demonstrated that $\beta$-catenin, a central figure in the pathway, is overexpressed in malignant gliomas [21]. The data that we have presented indicating a role for NARF in promoting cell growth or viability seem to be in conflict with the previous report that NARF is a negative regulator of the Wnt- $\beta$-catenin signaling pathway. Two possible explanations exist to reconcile this difference. First, while in general this pathway is believed to promote cell growth and tumor progression, there are transcriptional targets of the TCF/LEF family transcription factors that function as tumor suppressors. For example, members of the Dickkopf (Dkk) family of proteins all function to suppress the growth of several human cancers [22-24]. Dkk-3 has been shown to induce cell death in malignant gliomas [25]. So, it is possible that NARF functions as a negative regulator of atypical Wnt signaling, which would otherwise lead to tumor suppression. A second and perhaps more likely possibility is that NARF functions in 
one or more distinct pathways outside of the canonical Wnt pathway. Gene expression experiments including those within the Gene Expression Atlas show a high expression of NARF in testis, an organ known for its high rate of cellular proliferation. It is possible that NARF functions to promote cell division by way of another cellular mechanism or signaling pathway.

Our data are the first to demonstrate a potential role for this novel ubiquitin ligase in cell division, cell viability, or the tumorigenic process. While we do not yet know the exact mechanism by which NARF is exerting its effects, these data provide a foundation for future exploration into this protein and its role in cancer.

\section{REFERENCES}

1. Weissman, A. M. (2001) Nat Rev Mol Cell Biol, 2, 169-78.

2. Deshaies, R. J. and Joazeiro, C. A. (2009) Annu Rev Biochem, 78, 399434.

3. Lorick, K. L., Jensen, J. P., Fang, S., Ong, A. M., Hatakeyama, S. and Weissman, A. M. (1999) Proc Natl Acad Sci U S A, 96, 11364-9.

4. Chen, Z. J. and Sun, L. J. (2009) Mol Cell, 33, 275-86.

5. Sun, L. and Chen, Z. J. (2004) Curr Opin Cell Biol, 16, 119-26.

6. van Wijk, S. J. and Timmers, H. T. (2010) Faseb J, 24, 981-93.

7. Yamada, M., Ohnishi, J., Ohkawara, B., lemura, S., Satoh, K., HyodoMiura, J., Kawachi, K., Natsume, T. and Shibuya, H. (2006) J Biol Chem, 281, 20749-60.

8. Giannini, A. L., Gao, Y. and Bijlmakers, M. J. (2008) Biochem J, 410, 101-11.

9. Iuchi, S. (2001) Cell Mol Life Sci, 58, 625-35.

10. Fisher, R. D., Wang, B., Alam, S. L., Higginson, D. S., Robinson, H., Sundquist, W. I. and Hill, C. P. (2003) J Biol Chem, 278, 28976-84.

11. Zhao, H., Li, C. C., Pardo, J., Chu, P. C., Liao, C. X., Huang, J., Dong, J. G., Zhou, X., Huang, Q., Huang, B., Bennett, M. K., Molineaux, S. M., Lu, H., Daniel-Issakani, S., Payan, D. G. and Masuda, E. S. (2005) J Immunol, 174, 5288-97.
12. Arimoto, K., Takahashi, H., Hishiki, T., Konishi, H., Fujita, T. and Shimotohno, K. (2007) Proc Natl Acad Sci U S A, 104, 7500-5.

13. Capon, F., Bijlmakers, M. J., Wolf, N., Quaranta, M., Huffmeier, U., Allen, M., Timms, K., Abkevich, V., Gutin, A., Smith, R., Warren, R. B., Young, H. S., Worthington, J., Burden, A. D., Griffiths, C. E., Hayday, A., Nestle, F. O., Reis, A., Lanchbury, J., Barker, J. N. and Trembath, R. C. (2008) Hum Mol Genet, 17, 1938-45.

14. Margareto, J., Leis, O., Larrarte, E., Idoate, M. A., Carrasco, A. and Lafuente, J. V. (2007) J Mol Neurosci, 32, 53-63.

15. Sambrook, J. and Russell, D. (2001) Molecular Cloning: A Laboratory Manual, $3^{\text {rd }}$. ed.

16. Crawford, D. F. and PiwnicaWorms, H. (2001) J Biol Chem, 276, 37166-77.

17. Lo, P. K., Chen, J. Y., Lo, W. C., Chen, B. F., Hsin, J. P., Tang, P. P. and Wang, F. F. (1999) Oncogene, 18, 7765-74.

18. Ma, Y. X., Zhang, S. Z., Hou, Y. P., Huang, X. L., Wu, Q. Q. and Sun, Y. (2003) Sheng Wu Hua Xue Yu Sheng $\mathrm{Wu} W u$ Li Xue Bao (Shanghai), 35, 230-7.

19. Barker, N. (2008) Methods Mol Biol, 468, 5-15.

20. Gavert, N. and Ben-Ze'ev, A. (2007) $J$ Cell Biochem, 102, 820-8.

21. Liu, X., Wang, L., Zhao, S., Ji, X., Luo, Y. and Ling, F. (2010) Med Oncol.

22. Niehrs, C. (2006) Oncogene, 25, 7469-81.

23. Chen, J., Watanabe, M., Huang, P., Sakaguchi, M., Ochiai, K., Nasu, Y., Ouchida, M., Huh, N. H., Shimizu, K., Kashiwakura, Y., Kaku, H. and Kumon, H. (2009) Int J Mol Med, 24, 789-94.

24. Zhou, X. L., Qin, X. R., Zhang, X. D. and Ye, L. H. (2010) Acta Pharmacol Sin, 31, 202-10.

25. Mizobuchi, Y., Matsuzaki, K., Kuwayama, K., Kitazato, K., Mure, $\mathrm{H}$., Kageji, T. and Nagahiro, S. (2008) Neuro Oncol, 10, 244-53. 


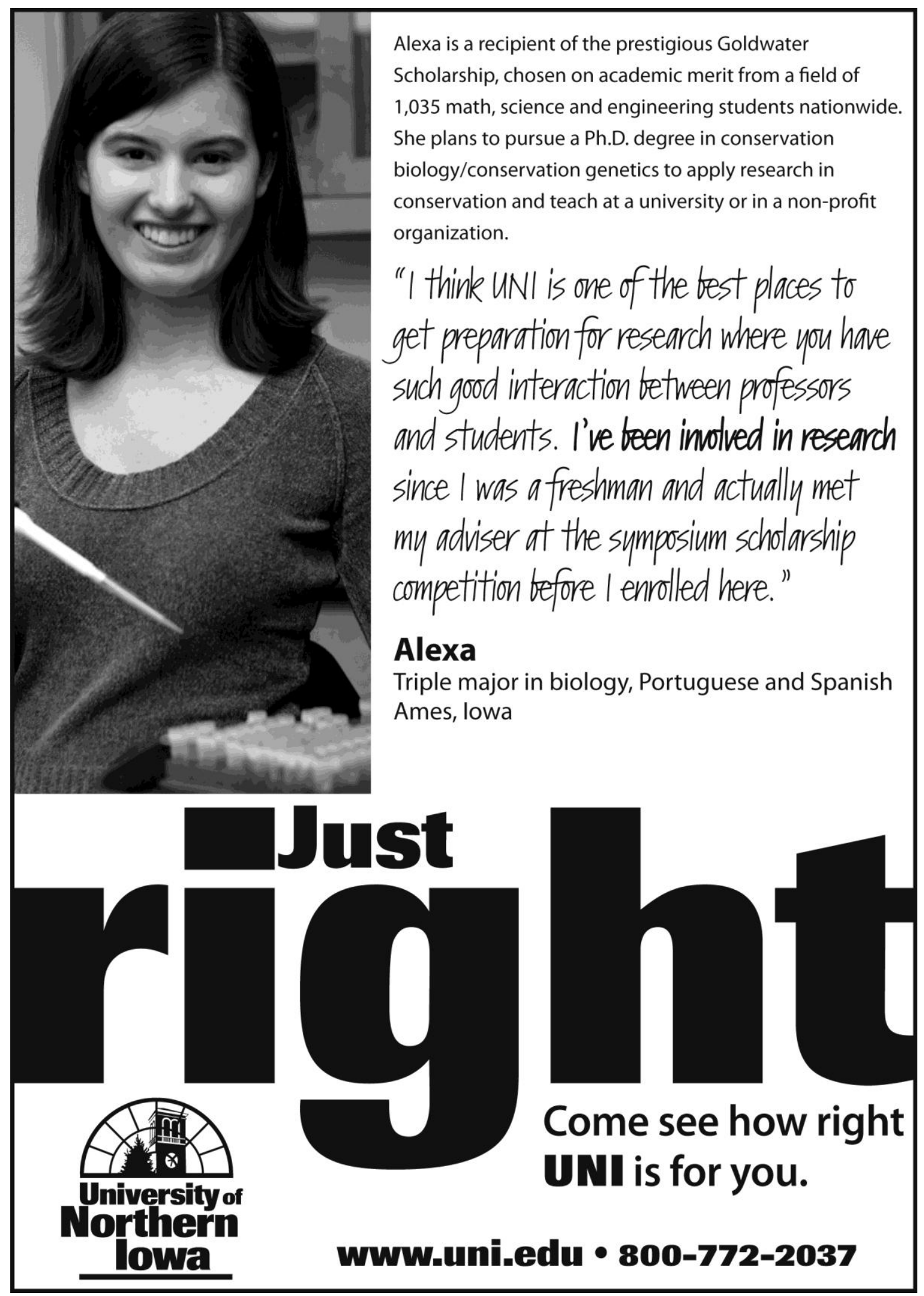




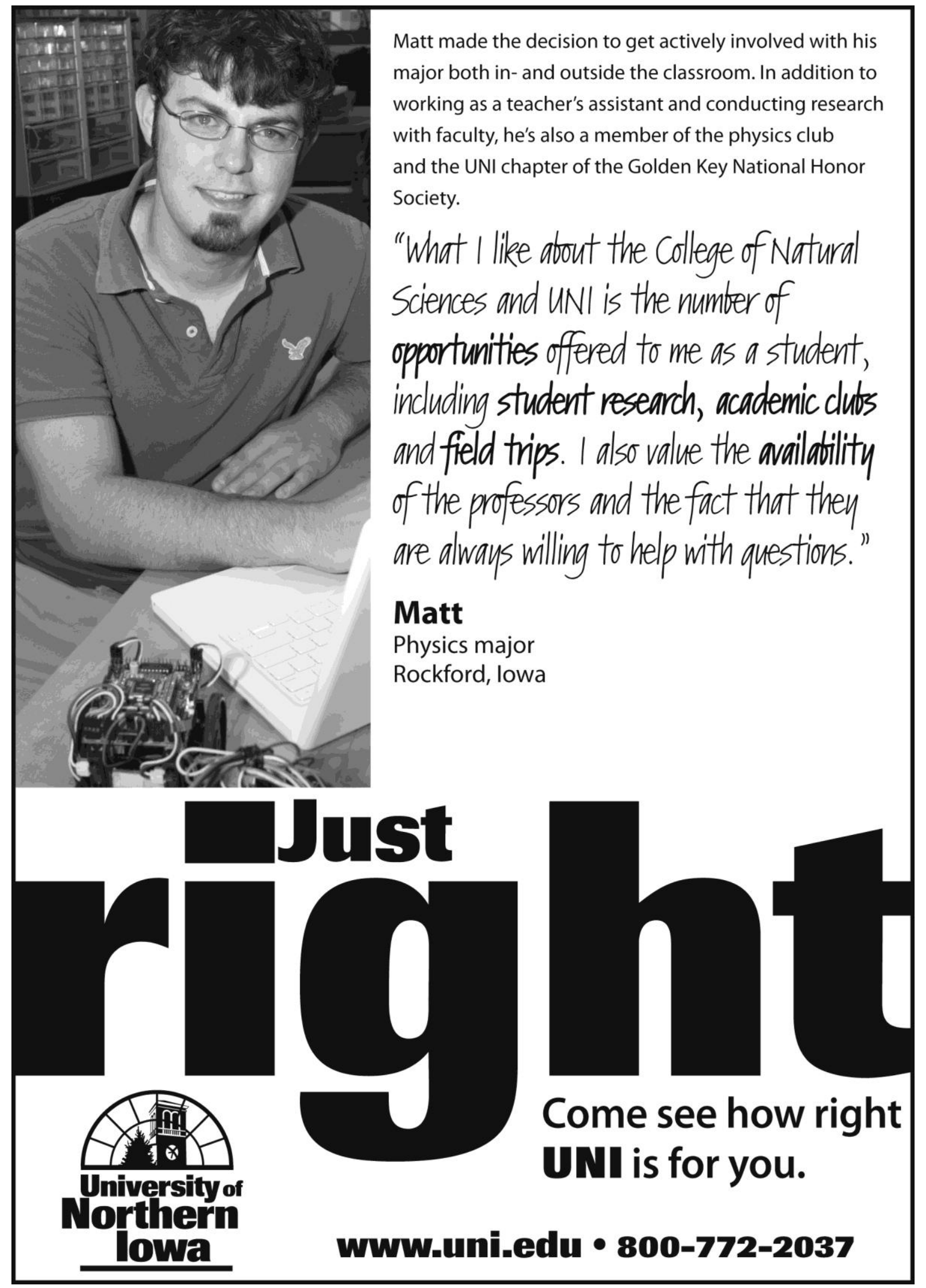

